\title{
Lineamientos para optimizar y fortalecer en el largo plazo la gestión de una entidad o política pública
}

Guidelines for optimizing and strengthening the management of an entity or public policy in the long term

\section{Héctor Briones}

\section{Universidad de Chile}

\section{Introducción}

El enfoque que se presenta a continuación está basado en los hallazgos y conclusiones de un estudio de caso, a partir del cual, se establecen y fundamentan lineamientos a considerar para optimizar y fortalecer en el largo plazo la gestión de una entidad o política pública en general.

Generalmente la institución, agencia, programa o política pública, preexiste a nuestra llegada, por lo tanto, es necesario empezar conociendo las definiciones básicas en relación a las acciones y/o productos de la institución o política pública. De igual forma, es necesario saber, qué plan de largo plazo sigue, según qué requerimientos y de cuántas entidades fiscales o ciudadanas cuenta para ello. Es inevitable plantearse que las posibilidades o caminos a seguir depende de cómo se ha entendido la definición de su quehacer a través del tiempo, pues ellas proporcionan la motivación espiritual de la organización, las respuestas a preguntas claves que surgen durante el proyecto.

Cuando hablamos de gestión de una agencia, programa o política pública, por lo general, olvidamos la influencia de las organizaciones e institucionalidad en los resultados esperados, y nos enfocamos en las formas de financiamiento, planificación, programas de trabajo, eficiencia y eficacia de la inversión y principalmente en asegurar la ejecución presupuestaria en el tiempo acordado.

La gestión actual se enfoca en implantar, soluciones probadas en otras agencias o incorporando software de última generación, dejando el estudio y fortalecimiento de la organización de la agencia relega a un segundo plano. En torno a la gestión se ha consolidado una industria que crea y ofrece modelos, protocolos, herramientas y servicios tendientes a resolver los problemas de las instituciones y personas que participan en ellas, sin embargo el contexto institucional varía de un país a otro, incluso dentro de un país de una zona a otra, por

*Dirección de correspondencia [Correspondence address]: Héctor Briones, Universidad de Chile E-mail: hectorbrionesp@gmail.com lo tanto, un alto porcentaje de esos proyectos, no tiene los resultados esperados. Estos modelos, protocolos, manuales y/o guías, aunque abundan, son de carácter técnico teórico, descriptivos, que si bien recogen directrices y lineamientos, generalmente se apartan u olvidan los principios y valores de los cuales provienen, dejando a los funcionarios sin herramientas para estimar los impactos proyectados y los no deliberados.

A través del estudio de caso "Institucionalidad para la Gestión del Mantenimiento Vial; caso chileno" (Briones, 2014) y sus conclusiones, se sugiere utilizar los principales hallazgos y conclusiones para que sean aplicables a otras organizaciones tanto públicas como privadas.

Este artículo muestra el caso del mantenimiento vial con dos objetivos, primero: introducir al lector en una agencia que durante los últimos 15 años ha tenido un aumento creciente de los presupuestos asignados, pasando de US\$644 millones en 1999 a US\$1.844 Millones en 2013, lo que habla de un trabajo constante del organismo en transmitir a las autoridades políticas la importancia de sus acciones y/o resultados y como han tomado consciencia de ello en los gobiernos. Segundo: a medida que se presentan los antecedentes se pretende que el lector los analice con el objetivo identificarlos y relacionarlos, lo más cercano posible, a una entidad o política pública que requiera su intervención.

\section{Metodología}

En el estudio de caso del Mantenimiento vial chileno (Briones, 2014), se utilizó un enfoque cualitativo, basado en entrevistas personales de manera de obtener las perspectivas y puntos de vista de los principales tomadores de decisión de la agencia vial chilena. El instrumento escogido para recolección de datos fue la entrevista semi-estructurada y revisión de documentos y literatura de manera que esta sea útil para complementar los planteamientos. Es importante señalar que entre lo señalado por la literatura y los datos emergentes de las entrevistas, los últimos tuvieron prioridad.

Al efectuarse sobre un tema poco conocido, el 
estudio se planteó a nivel exploratorio, de manera que la investigación permitiera obtener nuevos datos que conduzcan a formular una mejor reseña del mantenimiento vial en Chile. De igual forma tiene características descriptivas al procurar, a través de los entrevistados, conocer situaciones, costumbres, actitudes, formas de resolver y actuar que permitan caracterizar al mantenimiento vial en Chile. Finalmente el estudio se vuelve explicativo una vez que se comienzan a analizar los datos, identificar temas emergentes y dar cuenta de la realidad a través del material textual analizado.

En términos de etapas del desarrollo de la investigación, el trabajo fue construido en cinco momentos: En una primera instancia, se realizó un análisis bibliográfico y documental que contempló la revisión de la literatura en materia de mantenimiento vial en Chile y otros tres países, Colombia, España y Nueva Zelandia. Esto permitió, por un lado, generar los antecedentes de la investigación y, por otro, aproximar las dimensiones y preguntas guías que orientaron el trabajo de campo. En un segundo momento, se procedió al levantamiento de los datos de la investigación, por medio de la aplicación de entrevistas semi estructuradas a actores claves en la gestión del mantenimiento vial en Chile.

En la tercera etapa, se realizaron las transcripciones y se procedió a analizar el contenido de los textos, agrupando y categorizando las respuestas en temas y subtemas. La cuarta etapa consistió, a través de los temas y subtemas abordados por los entrevistados, en responder las interrogantes planteadas en el estudio. Finalmente al tener el punto de vista basado en las respuestas de los entrevistados a los ámbitos y objetivos del estudio e integrarlos con el análisis bibliográfico y/o documental, permitió arribar los principales resultados, elaborar las conclusiones y nutrir las propuestas de política pública que se presentan en la investigación.

Se aplicó una entrevista de corte semi estructurado a 7 directivos del primer nivel de decisiones relacionadas directamente con la gestión del mantenimiento vial, jefaturas en la agencia vial, jefes de unidades técnicas, consultores y jefatura de agencia relacionada, con el fin de obtener una visión que combinara las miradas de la dirección, la visión de jefaturas técnicas, la de consultores y contratistas, y la de agencias relacionadas.

Las entrevistas fueron movilizadas con el objetivo de capturar la percepción de los expertos en una serie de dimensiones de investigación o ámbitos de estudio: 1) Diagnóstico de la situación actual; 2) Relación con actores relevantes; 3) Descentralización y rol de las regiones; 4) Factibilidad de un modelo tipo agencia; y 5) Recomendaciones para la gestión del Mantenimiento vial.

\section{La evolución del modelo chileno de mantenimiento vial}

En 1970 la Red Vial Chilena poseía un adecuado estado para esa época. Pero, a partir de 1971 comienza a sufrir un continuo y severo deterioro, provocado principalmente por mantenimiento insuficiente y un uso mayor al previsto, situación similar para muchos países en desarrollo en aquella época, que habían ampliado la red de transporte más rápidamente que los presupuestos de mantención y capacidad institucional.

En 1977 se presentan numerosos deterioros en la capa de rodadura de la Ruta 5 o Carretera Panamericana en distintos puntos del país, construida principalmente en hormigón. Estos hechos llevaron a plantear la necesidad de conservar lo que tanto tiempo y recursos había costado construir y que por falta de mantenimiento se estaba perdiendo. Ese año se toma consciencia de la importancia de mantener los caminos, se cambian las concepciones y formas de trabajar para lograr aumentar los puntos de intervención y alcanzar mayores $\mathrm{km}$ mantenidos. Se comienza a delegar cada vez más la ejecución de trabajos a empresas privadas, lo cual ha evolucionado hasta hoy, donde no sólo se licita la ejecución sino que también el diseño de carreteras bajo la supervisión de los departamentos respectivos del nivel central.

La longitud de la Red Vial Chilena, a cargo de la Dirección de Vialidad (DV) del Ministerio de Obras Públicas (MOP) no ha sufrido grandes alteraciones durante las últimas décadas, manteniéndose en alrededor de los $80.000 \mathrm{~km}$. Sin embargo, la composición de la red ha presentado notorias variaciones, pasando de un $12 \%$ de red vial pavimentada en el año 1980 a un $23 \%$ en el año 2012.

Como se ha señalado, en el año 1977 se decide revertir el crítico deterioro de la Red Vial; para lo cual se plantea restablecer, expandir y consolidar el mantenimiento vial, desarrollar y modernizar la DV, por lo cual se establecen préstamos con el Banco Interamericano de Desarrollo (BID) y el Banco Mundial (BIRF).

Hasta 1985, los préstamos del Banco Mundial para el sector vial de Chile se destinaron principalmente a proyectos específicos de construcción. Pero, debido a su rápido aumento, Chile ya no podía atender las necesidades de mantenimiento con los fondos centrales y los mecanismos institucionales descentralizados. Desde 1975 la responsabilidad del $70 \%$ 
(56.000 km) de la red vial recaía sobre los gobiernos municipales, que carecían de los recursos técnicos, administrativos y financieros para poder cumplirla. Ese año, el organismo central de vialidad decidió volver a asumir gradualmente la responsabilidad de los caminos locales y utilizar contratistas privados, siempre que éstos fueran competitivos. Asimismo, se introdujo el sistema de planificación integral del sector, utilizándose para ese fin el Modelo del Banco Mundial para Normas de Diseño y Mantenimiento de Carreteras. Se decidió también aumentar el presupuesto central para mantenimiento vial de US\$78 millones en 1986 a US\$123 millones en 1988, así como reasignar dos tercios del presupuesto de inversión a actividades de rehabilitación y reconstrucción (US\$44 millones al año). A fin de respaldar la adopción de estas medidas y el programa de inversiones viales y mantenimiento para 1986-88, el Banco Mundial aprobó la concesión al país de un préstamo sectorial de US\$140 millones. El desembolso de los fondos del préstamo estuvo vinculado a la realización de las actividades de mantenimiento a fin de asegurar la ejecución oportuna de este prioritario programa (Mundial, 1988).

El financiamiento del mantenimiento vial en los últimos 30 años ha pasado por tres periodos. El primero entre los años 1982 a 1989 en el que se produce un aumento paulatino de los presupuestos de la DV, con un importante financiamiento internacional. El segundo periodo comprende entre 19901998 , en el cual se produjo un aumento creciente de los presupuestos de la DV, con gran aumento inicial, entre 1990 a 1995 y luego una fuerte reducción hasta hoy del financiamiento internacional. La tercera etapa entre el 2000 y 2013 se ha caracterizado por un aumento constante del presupuesto de la DV y ausencia de financiamientos internacionales. En la actualidad el financiamiento de la DV, depende directamente del Ministerio de Hacienda y forma parte del presupuesto nacional asignado cada año al Ministerio de Obras Públicas (Delgado, 2008).

En 1978 se inicia la rehabilitación, con el financiamiento internacional, de los $3.800 \mathrm{~km}$. de la principal vía del país, denominada Ruta 5, cuyo condición pasa de un $10 \%$ en estado bueno en el año 1978 a un de $87 \%$ en buen estado en el año1989. Tras la recuperación de la Ruta 5, se comienza a trabajar con más fuerza en la rehabilitación del resto de la red.

Hasta inicios de los años 80, sólo existía la modalidad de mantenimiento por "Administración Directa", aún vigente. En 1982 se implementa la modalidad de "Contratos a Precios Unitarios", en el cual las Direcciones Regionales de Vialidad contratan, con empresas contratistas, operaciones de manteni- miento rutinario y periódico de caminos o tramos de caminos, a precios unitarios y por un período de 6 a 9 meses.

En 1990 se crea el "Departamento de Gestión Vial" que anualmente efectúa un catastro de los tipos de capas de rodadura y bianualmente realiza una inspección visual de la red pavimentada, la que combinada con mediciones de IRI (International Roughness Index) y conteos de tránsito, permite determinar el estado de las capas de rodadura.

En 1992 la DV implementa a nivel nacional el "Sistema de Administración del Mantenimiento" (SAM) un conjunto de procedimientos administrativos y técnicos cuyo objetivo es lograr un mantenimiento eficiente de la Red Vial con la modalidad de Administración Directa.

En 1992, se agrega la modalidad de "Contratos Globales de Redes Viales". Las operaciones, definidas en cuanto a tipo y cantidades de obras, se contratan a serie de precios unitarios, comprendiendo también ítems eventuales para operaciones de emergencia. La experiencia con los "Contratos Globales" demostró la conveniencia de poder ofrecer al contratista la oportunidad de efectuar innovaciones tecnológicas o de materiales para mejorar la ejecución y el resultado de las operaciones y con ello asegurar a los usuarios un buen nivel de servicio.

En diciembre de 1993 se promulgó la Ley 19.265, y en Junio de 1994 el Decreto MOP N²14 con el reglamento, que permite a la Dirección de Vialidad comprar maquinaria destinada a la conservación de caminos y entregarla en comodato a las "Corporaciones Viales" sin fines de lucro, con las cuales se esperaba promover la participación organizada de entidades públicas y privadas y especialmente de la comunidad en la tarea del mantenimiento vial comunal, sin embargo esta iniciativa no tubo los resultados esperados, la utilización de los equipos no fue eficiente debido a que no lograron, de parte de las Municipalidades y/o de la comunidad en general, el apoyo económico mínimo para el funcionamiento normal, dado que atendían a una red de caminos que correspondían a sectores de bajos recursos que es donde se encuentran los caminos comunales secundarios.

El años1995 surgen los "Contratos por Nivel de Servicio", orientados a la red pavimentada y en los cuales el contratista asume por 5 años plena responsabilidad sobre una red definida y la DV se encarga de verificar que en ella exista permanentemente el nivel de servicio licitado. Lamentablemente con esta modalidad de mantenimiento sólo se adjudicaron dos contratos con espléndidos resultados, pero las crisis económicas de los años subsiguientes impidie- 
ron continuar.

En el año 2000 se publica el Volumen N7, "Mantenimiento Vial" del Manual de Carreteras de la DV, documento que comprende los aspectos relativos a la gestión, programación, inspección y procedimientos, técnicos y administrativos, que se utilizan en el mantenimiento de la red vial, cualquiera sea la modalidad administrativa o forma de contrato en que ésta se desarrolle.

El año 2008 se integra otra modalidad de conservación, los "Contratos de Conservación Global Mixtos por Nivel de Servicio y Precio Unitario", que tienen una duración de cinco años y contemplan el mantenimiento de una parte de la infraestructura vial bajo la modalidad de Conservación Global a Precios Unitarios y la otra por Niveles de Servicio a Suma Alzada (oferta a precio fijo con las cantidades de obras inamovibles).

Desde el 2008 la DV ha trabajado el perfeccionamiento de los mecanismos jurídicos, y económicos para la implementación de una nueva modalidad, que se postergó, debido al cambio conceptual desde un contrato de mantenimiento de largo plazo por niveles de servicio, a una concesión propiamente tal, regida por la ley de concesiones. Hoy el "Programa de Concesión de Mejoramiento, Rehabilitación y Conservación de Redes Viales Regionales", es liderado por la Coordinadora de Concesiones del MOP con la finalidad de introducir en el mercado una nueva forma de conservar los caminos públicos del país, utilizando los mecanismos jurídicos, financieros y económicos que establece la Ley de Concesiones de Obras Públicas.

En forma paralela y con asistencia técnica del Banco Mundial y financiamiento parcial de una donación del Japón, se iniciaron en 1990 los estudios y las acciones legislativas necesarias para poner en marcha un "Programa de Concesiones Viales", lo que se concretó en concesiones viales con un largo total de $2385 \mathrm{~km}$ desde 1994 hasta el 2012.

El año 2009 en el marco del Convenio de Desempeño para la Alta Dirección Pública, el Director de Vialidad comprometió la elaboración de una política de conservación vial en conjunto con la Dirección de Planeamiento del MOP, cuyo alcance fue establecer para los diferentes tipos de capas de rodadura, un grupo de acciones de conservación a ser realizadas en función de umbrales admisibles y comenzar a implementar con gradualidad planes regionales de conservación. El amplio alcance del establecimiento de esta política hizo necesario efectuar el desarrollo del trabajo en tres etapas: Etapa 1, para caminos pavimentados, desarrollado y finalizado el año 2010. Etapa 2, para caminos básicos, desarrollado y finalizado el año 2011 y Etapa 3, para caminos ripiados, trabajo desarrollado durante el año 2012.

Actualmente y como siguiente paso se trabaja en la implementación gradual de la política planteada, analizando y evaluando los efectos sobre el estado de las capas de rodadura, reconociendo las dificultades y proponiendo las mejoras necesarias.

Paralelamente la Sub Dirección de Mantenimiento desde 2007 está trabajando en el Proyecto SGM - Sistema de Gestión del Mantenimiento, que se enfoca en el mejoramiento de la Planificación de la Conservación mediante la aplicación de un modelo de gestión, que comenzó a implementarse el 2011 en las Regiones de Arica - Parinacota, Valparaíso y Los Ríos, con el módulo para el registro de la información de Conservación por Administración Directa (CAD) del Sistema FEMN ${ }^{1}$, plataforma informática que soporta el modelo de gestión, abarcando actualmente todas las regiones del país. Lo anterior, implica la generación de órdenes de trabajo (OT) desde el sistema y el registro de la información de la ejecución de dichas órdenes, además de manera complementaria, también entró en operación el módulo para el registro de las Bitácoras ${ }^{2}$ para los equipos productivos.

Como se puede apreciar, la estructura actual de gestión de mantenimiento de la red vial nacional es el resultado de años de sucesivas adaptaciones de la agencia vial, para responder a los requerimientos de distintas instituciones del país y de las autoridades políticas respecto del cumplimiento del gasto, siendo la estructura actual totalmente funcional para el consecución efectiva de este indicador.

Este perfeccionamiento constante del sistema de gestión del mantenimiento chileno, ha cedido poco a poco la planificación y ejecución de las obras físicas, desde el nivel nacional al regional, en la medida en que las regiones han contado con disponibilidad de recursos, capacidades técnicas y política, forjando de esta forma la experiencia que hoy tienen.

\section{Resultados}

\section{Gestión Estratégica}

Se constató que la agencia vial no tiene una visión formalizada y la misión posee tres interpretaciones

\footnotetext{
${ }^{1}$ Sistema FEMN: Plataforma computacional integrada y en línea (MAXIMO-IBM), para la administración de la información de Conservación. El nombre del sistema es FEMN, concepto en mapudungun que significa "Hacer bien las cosas".

${ }^{2}$ Bitácoras: Planilla para el registro de la información de utilización de los equipos productivos y vehículos.
} 
sobre qué mantener (el estándar de los caminos, el patrimonio vial y la conectividad). Los entrevistados al ser consultados señalan al indicador del estado del camino, como el principal indicador para la gestión del mantenimiento vial. Sin embargo en las acciones el factor más influyente es el gasto presupuestario. Para los directivos una buena gestión, es eficacia en la ejecución presupuestaria.. La agencia vial mide indicadores, pero no forman parte de ningún plan de metas y objetivos, ni de la agencia ni de sus directivos, de esta forma la misión no tiene indicador.

En la labor de mantenimiento, se reconocieron tres fases, la primera es de planificación, fase en que las oficinas regionales se apoyan en el nivel central por carencia de personal técnico especializado y porque la inversión de montos superiores es una decisión que se toma a nivel central. La fase de ejecución es asumida totalmente a nivel regional. La fase de continuidad aparece por la importancia que toma para los entrevistados la planificación de contratos para evitar que existan brechas entre término de uno y comienzo de otro. Además, si bien la restricción presupuestaria es una debilidad, esta es conocida y una situación a nivel mundial, si bien no hay presupuesto para logra atender todo, se atiende lo urgente y se priorizan las intervenciones.

\section{Estructura de la Agencia}

La estructura actual de gestión de mantenimiento de la red vial nacional de Chile es resultado de años de sucesivas adaptaciones surgidas de tal forma de responder a cúmulo de requerimientos, presentados algunos por el Ministerio de Hacienda, otros debido a implementación de políticas gubernamentales o adaptaciones a nuevas metodologías para mejor uso de los recursos, incorporación de nuevas técnicas y tipos de contratos y/o dar respuestas a petitorios de autoridades locales, etc.. Con todo, sin lugar a dudas el principal catalizador de los cambios ha sido el cumplimiento del gasto, siendo la estructura actual totalmente funcional para el cumplimiento efectivo de este indicador.

Hoy la estructura de la agencia vial para la gestión del mantenimiento de la red vial de Chile, funciona muy bien en pos del indicador principal, alcanzando un gasto del presupuesto del $100 \%$ en los últimos dos años.

Existe unanimidad en los entrevistados respecto a la eficacia de la Administración Directa en las emergencias y ese hecho justifica su existencia, sin embargo se agrega que también permite realizar conservaciones en lugares de difícil acceso o donde la empresa privada no interviene por ser poco atractivo económicamente.

La evolución endógena del sistema de gestión del mantenimiento vial chileno, es un sistema que poco a poco ha delegado la planificación y ejecución de los mantenimientos, desde el nivel nacional al regional. Sin embargo, la centralización administrativa limita la autonomía del nivel local. Los Directores Regionales actúan por delegación administrativa de las funciones del Director Nacional, sin tener responsabilidad directa por las acciones delegadas. A pesar de la posibilidad que tienen las agencias locales de concebir sus planes de trabajo, no cuentan con independencia formal para ello, ni financiera ni administrativa.

La red de caminos nacionales declarados públicos es única, de aproximadamente $77.764 \mathrm{Km}$, y está a cargo de la Dirección de Vialidad, agencia de carácter nacional que opera con oficinas regionales. Gran parte de los entrevistados está de acuerdo en la conveniencia de entregar la tuición de las redes secundarias y terciarias a los gobiernos locales o comunidad organizada, dejando sólo los caminos de mayor tránsito a la Dirección de Vialidad. Claramente el traspaso de la tuición debe ser acompañada por recursos financieros y técnicos para realmente tener capacidad de administrar esas redes. La existencia de agencias viales regionales y su autonomía, se plantea como una decisión de política de gobierno y no de la agencia vial. Si bien actualmente el sistema de gestión del mantenimiento vial se apoya en las Direcciones Regionales, estas no existen administrativamente y actúan solo por delegación del Director Nacional en ejercicio y tienen las atribuciones que él determina. Cuando se habla de agencias regionales autónomas, los entrevistados piensan en algo parecido a los SERVIU ${ }^{3}$ donde estas agencias serían órganos independientes creados por ley, con patrimonio y presupuesto propio.

\section{Gestión de Personal}

Las principales fortalezas del sistema de mantenimiento se encuentran en los recursos humanos y en la consciencia de las autoridades respecto a la importancia del mantenimiento vial, reflejado en los presupuestos asignados, los que se han incrementado en las últimas décadas. Además ha tenido gran

\footnotetext{
${ }^{3}$ Los Servicios de Vivienda y Urbanización son Instituciones Autónomas del Estado, relacionadas con el Gobierno a través del Ministerio de Vivienda y Urbanismo, con personalidad jurídica de derecho público, con patrimonio distinto del Fisco, y de duración indefinida. Pueden utilizar la sigla "SERVIU" para su denominación, agregando la mención relativa a la región correspondiente, se rigen por el DS N³55 del MIMVU de 1976 que aprueba su reglamento orgánico.
} 
impacto en la gestión de la agencia vial la incorporación al Sistema de Alta Dirección Pública la elección mediante concurso público de los directivos de primer y segundo nivel jerárquico, los que tienen una duración de tres años en su cargo y pueden ser renovados hasta dos veces, por igual periodo. Tienen dedicación exclusiva y firman un convenio de desempeño, instrumento de gestión que cumple con la doble función de orientar y evaluar.

\section{Gestión Financiera}

La restricción presupuestaria se plantea como una debilidad importante para el sistema. Un reflejo de ello es la escasez de inspectores fiscales. La capacidad técnica en regiones es aún limitada haciéndolas dependientes del nivel central. Actualmente el presupuesto para mantenimiento vial permite una cobertura para el $80 \%$ de la red vial nacional.

El financiamiento del mantenimiento de un camino es posible traspasarlo al usuario a través del cobro de peajes sólo en los que tengan un tránsito alto, de otra forma sale más caro el cobro del peaje que lo pagado por el usuario. La recaudación de impuestos específicos asignados al mantenimiento de caminos, es visto como ineficiente y un incentivo al gasto no racionalizado al tener financiamiento asegurado.

Sin embargo se ha avanzado en incorporar en el mantenimiento vial a los usuarios, a través del cobro de peajes, convenios con las empresas que utilizan mayormente el camino y/o gobiernos locales que realizan aportes de maquinaria para la administración directa. Con todo, el sistema de financiamiento actual está entregando un resultado de mantenimiento e inversión insuficiente. Se plantea avanzar hacia una gestión basada en el cobro directo al usuario para el mantenimiento de la vía, a través del empleo de tecnologías que permiten recaudar por uso del camino. Una forma indirecta de financiamiento ha surgido en los últimos años, de parte de los gobiernos regionales quienes usando recursos del Fondo Nacional de Desarrollo Regional (FNDR), han comprado y renovado maquinaria para la administración directa, sin embargo, los equipos necesitan operarios y gastos de combustible que no están contemplados en estas asignaciones del gobierno regional (DIPRES, 2014).

El financiamiento de la agencia vial proviene mayoritariamente del Gobierno Central. Si se pretende traspasar la tuición de sus caminos a las Direcciones Regionales o locales, es ineludible traspasarle los fondos necesarios para el mantenimiento de ellos. Respecto a cómo traspasar el presupuesto, también es motivo de análisis más profundo, sin embargo, en primera instancia se puede recomendar, conforme a la institucionalidad actual, utilizar FNDR y el ISAR con indicaciones de exclusividad de uso de recursos para mantenimiento vial, ya que en el pasado se traspasaron las redes terciarias, pero no se aseguraron los recursos para su mantenimiento, generándose el deterioro de los caminos. En el largo plazo es posible plantear un fondo vial, pero es una discusión que sugiere una restructuración de nivel institucional de la agencia y responsabilidad a nivel de gobierno

\section{Relación con Actores Externos}

Si bien no es mencionada por las jefaturas de primer nivel jerárquico en la agencia, la poca difusión de los criterios y políticas de mantenimiento, cobra importancia en la medida que nos alejamos de las jefaturas y nos acercamos a usuarios y agentes relevantes que interactúan con la agencia. El tema de la difusión de las políticas de mantenimiento, toma importancia en la medida que los agentes que interactúan con la agencia no conocen la política concreta que determina el mantenimiento vial, lo que obliga a aceptar a ojos cerrados proyectos planteados.

\section{Las Relaciones con otros ministerios, se reduce al Ministerio de Hacienda}

La Dipres (Dirección de Presupuesto del Ministerio de Hacienda) controla que los recursos destinados al mantenimiento se empleen en obras de mantenimiento, dejando a la agencia determinar cómo, dónde y cuándo actuar. La disminución y flexibilización de los controles administrativos es valorada en forma positiva sobre todo al plantearse que la actual forma de control, está basada en la confianza y en apoyar la autonomía y flexibilidad de los recursos en un área técnica y compleja que necesita de capacidad de reacción, espacio de operación para responder a las exigencias del día a día, agregando que cierto grado de control es necesario y conveniente.

\section{Conclusiones}

A continuación se utilizan los principales resultados hallazgos del caso del mantenimiento vial chileno para aplicarlos a otras organizaciones, estableciendo los principales conceptos a tener en cuenta para redefinir y estructurar la institucionalidad de una entidad o política pública. Luego se sugieren 
lineamientos para optimizar y fortalecer en el largo plazo la gestión de una entidad pública.

En los países en que se han realizado reformas significativas en la institucionalidad de las agencias, ellas han sido gatilladas por cambios originados fuera de la agencia y que corresponden a revisiones y conceptualizaciones respecto del Estado y su función.

La agencia o entidad debe discernir y consensuar la definición de su producto, lo que entenderá por obra (inversión) versus servicio. La obra implica la ejecución de un resultado determinado, que se traduce en una construcción de naturaleza inmueble. El servicio es una actividad como tal, es un medio para la obtención de un resultado. La claridad de los límites que se establezcan entre obra y servicio permitirá la elaboración de la misión con indicadores y umbrales, ayudando a orientar la toma de decisiones y los objetivos de los funcionarios de la agencia vial. La claridad frente a lo que se debe hacer y lo que se espera de la agencia, da luz para proyectar el tipo de organización y estructura acorde para cumplir con las expectativas. La Evolución Endógena del Sistema de gestión del Mantenimiento Vial Chileno es un buen ejemplo de ello.

La ausencia de visión sintetizada para la agencia allana el camino para la ausencia de objetivos a largo plazo, de bases para generación de prioridades basadas en acuerdos, en condiciones, y acciones que contribuyan a la misión y su verificación. El establecimiento de indicadores vinculados a la misión, entrega claridad y precisión en las metas y objetivos. Sin embargo, estos deben ser comprobables y medibles para dar cuentas por ellos, por eso es imprescindible establecer junto a los indicadores, sus respectivos umbrales de desempeño. Además, es necesario, debido a la restricción presupuestaria que limita la atención a todo lo que se requiere, la fijación de metas basadas en los indicadores.

La firma de convenios de desempeño, cumple con la doble función de orientar y evaluar; razón por lo cual, es indispensable incorporar las metas de desempeño de la agencia a los cargos, como parte primordial de las metas de gestión de los Directivos, fijando umbrales de cumplimiento durante su periodo de ejercicio por los cuales será remunerada y evaluada su gestión.

La información con acceso sencillo y oportuno son la base del sistema de transparencia y de la rendición de cuentas. Los flujos de información a través de los indicadores y publicación de un texto explicito con las políticas a implementar, son los puentes que vinculan a los distintos actores en el área de influencia de la agencia.
La reestructuración institucional de las agencias debe ir acompañada de transferencias de tuición desde el nivel nacional al nivel regional o local. Se llega a una agencia nacional que supervisa, unifica criterios y que tiene bajo su tuición la red principal del país; y a agencias locales a cargo de redes secundarias con gestión y presupuesto propio.

Se debe pensar en que la estructura actual de gestión de la entidad o política pública es resultado de años de sucesivas adaptaciones surgidas en las circunstancias de los tiempos, generados endógenamente. Lo más prudente es potenciar el sistema actual de evolución endógena, catalizando el proceso y llevándolo al próximo nivel natural, elevando la responsabilidad regional en la gestión de la entidad o política pública. El financiamiento de las entidades o políticas públicas proviene mayoritariamente del Gobierno Central. Si se pretende traspasar la tuición a las Regiones o autoridades locales, es ineludible traspasarle los fondos necesarios para ello.

\section{Recomendaciones}

Se sugieren lineamientos a tomar en cuenta para la implementación de mejoras en entidades públicas. Se debe generar un debate interno a nivel nacional respecto del rol y misión de la entidad pública. Se trata que la agencia logre establecer la construcción de una Visión Estratégica, coordinación para la implementación y métodos de monitoreo y evaluación.

Es necesario avanzar en la determinación y jerarquización de la red que atiende la agencia o política pública. Se sugiere asumir un nuevo paso en la evolución natural del sistema actual y traspasar la responsabilidad a las Regiones, elegir Directores Regionales mediante concurso de Alta Dirección Pública quienes tendrán Convenio de Desempeño basados en metas a cumplir con indicadores y umbrales de desempeño para la red a su cargo, fijados por el Director Nacional.

Si los resultados de los organismos no tienen consecuencia sobre el nivel de asignación del gasto que los mismos reciben, tampoco cambiará en profundidad la cultura pública y para lograr esto es necesario que el parlamento tome en cuenta los resultados (Tavares y Berretta, 2006). Actualmente el parlamento centra su foco en el gasto presupuestario como medida de eficiencia y objetivo a cumplir, se plantea que la entidad o política pública trabaje en establecer un indicador de desempeño que refleje la mejoría del bienestar social y el crecimiento de la inversión en esta área. 
Para poder tomar en cuenta los resultados, tanto para asignaciones como para incentivos en la agencia, estos se deben medir y haber sido especificados, por lo tanto la agencia debe avanzar hacia establecer o determinar niveles de desempeño, o explicitar estándares.

Se sugiere incorporar en la orgánica de las decisiones locales multicriterio, la creación de un consejo regional de planificación, que recoja las opiniones de la comunidad a través de representantes. Este consejo es propuesto como instancia formal de acercamiento de la agencia a la comunidad local involucrada, que apoye los procesos de trasparencia institucional de rendición de cuentas y/o "accountability".

En cuanto al sistema actual de financiamiento se sugiere analizar y estudiar la posible implementación de un sistema que otorgue personalidad jurídica, autonomía administrativa y un patrimonio propio a la agencia, lo que permite que no solo reciba aportes del Estado para el mantenimiento de la red, sino también aportes de otras instituciones y organizaciones, tanto públicas como privadas, que permitan generar alianzas y convenios.

\section{Referencias}

Briones, H. (2014). Institucionalidad para La Gestión del Mantenimiento Vial: Caso Chileno. Tesis de magíster, Universidad de Chile.

Delgado, A. (2008). Chile: Experiencia en el Mantenimiento Vial. Revista Routes/Roads, (339):4349.

DIPRES (2014). Presupuestos Históricos. Technical report.

Mundial, B. (1988). Deterioro de los Caminos en Países en Desarrollo. Washington, DC.

Tavares, M. y Berretta, N. (2006). Sistemas de Planificación Estratégica e Innovaciones Presupuestarias. 